\title{
Measurement of heavy-flavour production, correlations and jets with ALICE
}

\author{
Shingo Sakai ${ }^{\text {a1 }}$ for the ALICE collaboration \\ ${ }^{1}$ Univ. of Tsukuba, Faculty of Pure and Applied Sciences, 1-1-1 Tennodai Tsukuba Ibaraki 305-8571 Japan
}

\begin{abstract}
In this article, recent ALICE measurements of the $R_{\mathrm{AA}}$ and $v_{2}$ of electrons at mid-rapidity $(|y|<0.6)$ and muons at forward rapidity $(2.5<y<4)$, from semi-leptonic decays of heavy-flavour hadrons in $\mathrm{Pb}-\mathrm{Pb}$ collisions at $\sqrt{s_{\mathrm{NN}}}=5.02 \mathrm{TeV}$ are shown. The results of D-tagged jet production cross-section in pp collisions at $\sqrt{s}=7 \mathrm{TeV}$ are presented. In addition, the azimuthal correlation of $\mathrm{D}$ mesons with charged particles in $\mathrm{p}-\mathrm{Pb}$ collisions at $\sqrt{s_{\mathrm{NN}}}=5.02 \mathrm{TeV}$, and electrons from semi-leptonic decays of heavyflavour hadrons with charged particles in $\mathrm{p}-\mathrm{Pb}$ and $\mathrm{Pb}-\mathrm{Pb}$ collisions at $\sqrt{s_{\mathrm{NN}}}=5.02 \mathrm{TeV}$ are reported.
\end{abstract}

\section{Introduction}

Heavy-flavour (charm and beauty) production provides relevant information to investigate the strongly interacting medium, the Quark-Gluon Plasma (QGP), formed in heavy-ion collisions at ultrarelativistic energies. Heavy quarks are produced primarily in the initial hard partonic interactions, and they propagate through the QGP. During that propagation they are expected to interact with the constituents of the QGP. The key observables for understanding the interaction are the nuclear modification factor $\left(R_{\mathrm{AA}}\right)$ and azimuthal anisotropy $\left(v_{2}\right)$. The $R_{\mathrm{AA}}$ is sensitive to the energy loss of partons in the hot and dense QCD matter which occurs via radiative [1] and collisional [2] processes. The energy loss has been predicted to depend on mass and colour charge of partons. In this picture charm and beauty quarks are expected to suffer a smaller energy loss than light flavour quarks $(u, d$ and $s)$. The $v_{2}$ is sensitive to the transport properties of the medium. And it reflects the collective motion of heavy quarks in the medium at low $p_{\mathrm{T}}$ as well as the path-length dependence of the energy loss at high $p_{\mathrm{T}}$ in the non-central collisions. Further understanding of properties of heavy quarks in the QGP can be obtained by measurements of jets and the azimuthal correlation of particles originating from heavy flavours, which are sensitive to the possible modification of the fragmentation of heavy quarks. Such measurements in $\mathrm{pp}$ and $\mathrm{p}-\mathrm{Pb}$ collisions allow studies of jet properties, and address cold nuclear matter and collective effects of heavy quarks.

\footnotetext{
asakai.shingo.gw@u.tsukuba.ac.jp
} 


\section{Results}

\subsection{Heavy-flavour production in $\mathrm{pp}$ and $\mathrm{p}-\mathrm{Pb}$ collisions}

Figure 1 shows the transverse-momentum-differential cross section of charged jets containing a D meson in pp collisions at $\sqrt{s}=7 \mathrm{TeV}$. The $\mathrm{D}^{0}$ cross section is well described by calculations based on perturbative QCD (pQCD) [3]. The measurement of charm-jet production allows one to further test $\mathrm{pQCD}$ calculations for heavy-flavour production. The measured cross section is compared with the expectation from POWHEG + PYTHIA in the figure. The plot in the bottom panel shows the ratio of data and the calculation, and it was found that the cross section from the data and the calculations are in good agreement in the range $5<p_{\mathrm{T}}<30 \mathrm{GeV} / c$. In ALICE, heavy-flavour production has also been studied by measuring the azimuthal correlation between heavy flavours and charged particles. Figure 2 shows the azimuthal correlation of $\mathrm{D}$ mesons with charged particles in $\mathrm{p}-\mathrm{Pb}$ collisions at $\sqrt{s_{\mathrm{NN}}}=5.02 \mathrm{TeV}$. The correlation was measured in various intervals of $p_{\mathrm{T}}$ ranges for the $\mathrm{D}$ meson and its associated charged particles. In the azimuthal correlation, the near-side $(\Delta \varphi=0)$ correlation is sensitive to the fragmentation function of charm quarks. On the other hand, the away-side correlation $(\Delta \varphi=\pi)$ is sensitive to the properties of the recoil jet. In $\mathrm{p}-\mathrm{Pb}$ collisions, possible modifications of the jet properties could arise from cold nuclear matter effects, like parton-density shadowing or saturation [4], a Cronin-like enhancement [5] and energy loss [6] in the initial state. The correlation was compared with PYTHIA calculations with different tunings. The PYTHIA calculations are in good agreement with the measured azimuthal correlation of $\mathrm{D}$ mesons with charged partcles in various $p_{\mathrm{T}}$ regions. The agreement indicates that the modification of jets from charm is not significantly affected by the cold nuclear matter effects as well as from possible final-state effects.

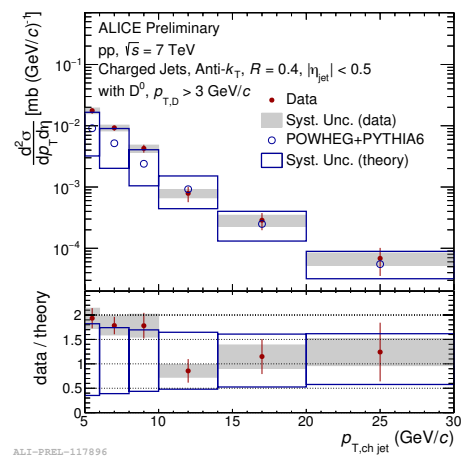

Figure 1: Transverse-momentumdifferential cross section of charged jets containing a $\mathrm{D}$ meson in $\mathrm{pp}$ collisions at $\sqrt{s}=7 \mathrm{TeV}$.

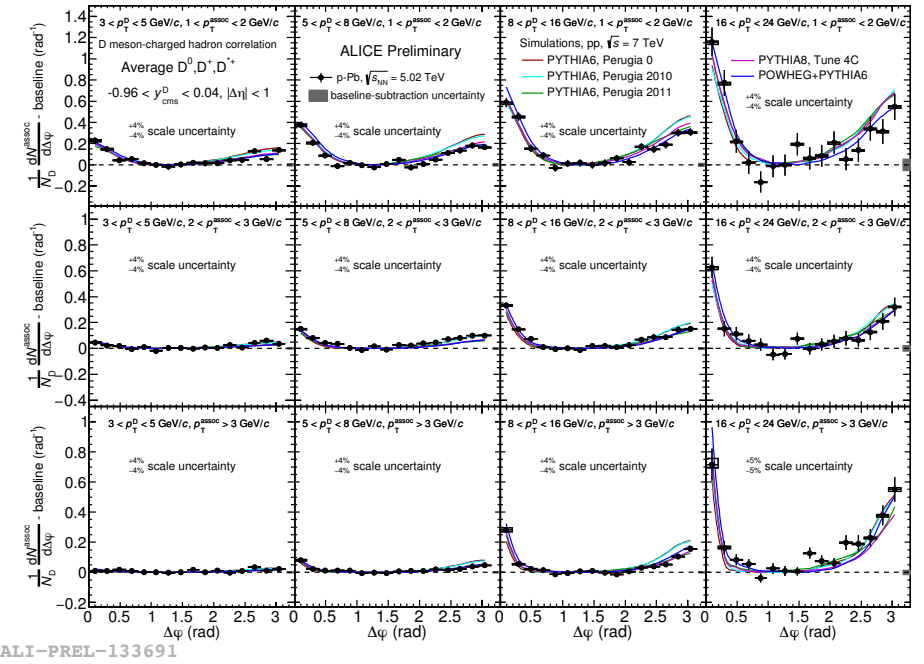

Figure 2: The azimuthal correlation of $\mathrm{D}$ mesons with charged particles in $\mathrm{p}-\mathrm{Pb}$ collisions at $\sqrt{s_{\mathrm{NN}}}=5.02 \mathrm{TeV}$.

\subsection{Heavy-flavour production in $\mathrm{Pb}-\mathrm{Pb}$ collisions}

The left panel of Fig. 3 shows $R_{\mathrm{AA}}$ of electrons from semi-leptonic decays of heavy-flavour hadrons $\left(e^{\mathrm{HF}}\right)$ at $|y|<0.6$ in $0-10 \%$ centrality in $\mathrm{Pb}-\mathrm{Pb}$ collisions at $\sqrt{s_{\mathrm{NN}}}=5.02 \mathrm{TeV}$. The result shows that 

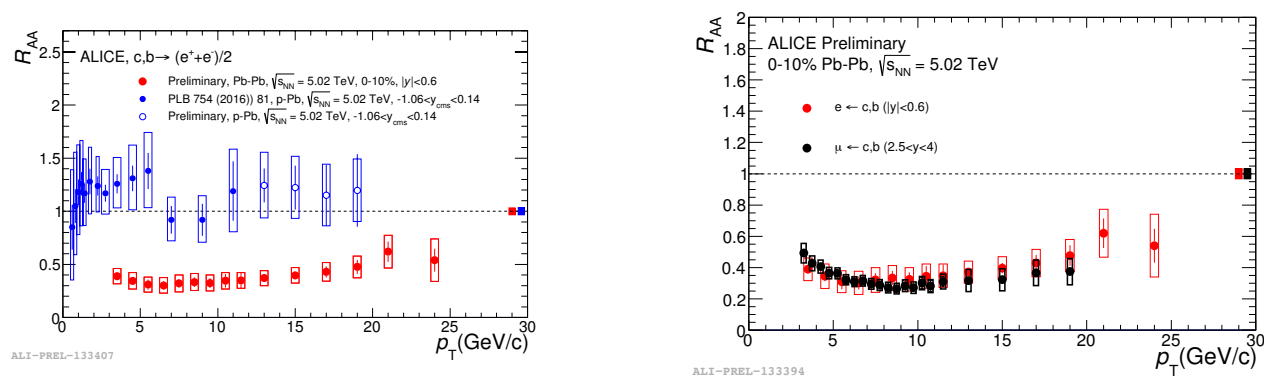

Figure 3: The nuclear modification factor for $e^{\mathrm{HF}}$ in $\mathrm{Pb}-\mathrm{Pb}$ and in $\mathrm{p}-\mathrm{Pb}$ collisions at $\sqrt{s_{\mathrm{NN}}}=5.02 \mathrm{TeV}$ (left), and of $e^{\mathrm{HF}}(|y|<0.6)$ and $\mu^{\mathrm{HF}}(2.5<y<4)$ in $0-10 \% \mathrm{~Pb}-\mathrm{Pb}$ collisions (right).

the $R_{\mathrm{AA}}$ is less than unity $\left(R_{\mathrm{AA}}<1\right)$ and it suggests that $e^{\mathrm{HF}}$ production is significantly suppressed up to $25 \mathrm{GeV} / c$. On the other hand, such strong suppression of $e^{\mathrm{HF}}$ is not observed in $\mathrm{p}-\mathrm{Pb}$ collisions at $\sqrt{s_{\mathrm{NN}}}=5.02 \mathrm{TeV}$. The nuclear modification factor of $e^{\mathrm{HF}}$ in $\mathrm{p}-\mathrm{Pb}$ collisions at $\sqrt{s_{\mathrm{NN}}}=5.02 \mathrm{TeV}$ is also shown in the Fig 3. It is consistent with unity within uncertainties, indicating that strong suppression observed in $\mathrm{Pb}-\mathrm{Pb}$ collisions is mainly due to energy loss of charm and beauty quarks in the hot medium formed in the collisions. A similar strong suppression is also observed in muons from heavy-flavour decays $\left(\mu^{\mathrm{HF}}\right)$ at forward rapidity $(2.5<\mathrm{y}<4)$ and the $R_{\mathrm{AA}}$ for $\mathrm{e}^{\mathrm{HF}}$ and $\mu^{\mathrm{HF}}$ are consistent in the range $3<p_{\mathrm{T}}<20 \mathrm{GeV} / c$ (Fig. 3, right). Further insights on heavy-flavour production in the hot and dense QCD matter can be obtained by measuring the per-trigger azimuthal correlation between $e^{\mathrm{HF}}$ and hadrons. Figure 4 shows the associated yield of charged particle in the near-side correlation peak in $0-20 \%$ and $20-50 \%$ central in $\mathrm{Pb}-\mathrm{Pb}$ collisions divided by the yield in $\mathrm{p}-\mathrm{Pb}$ collisions as a function of associated charged particles $p_{\mathrm{T}}$. The yield in $\mathrm{Pb}-\mathrm{Pb}$ collisions tends to increase at lower $p_{\mathrm{T}}$. Such an enhancement of associated charged hadrons at low $p_{\mathrm{T}}$ has been observed in the $\pi^{0}-h$ and $h-h$ correlations in $\mathrm{Pb}-\mathrm{Pb}$ collisions [7, 8]. In addition, a positive $v_{2}$ of $e^{\mathrm{HF}}$ has been observed

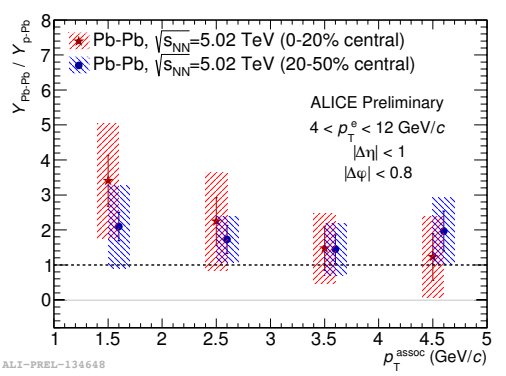

Figure 4: Ratio of near-side associated yields in $\mathrm{Pb}-\mathrm{Pb}(0-20 \%$ and $20-50 \%)$ and in $\mathrm{p}-\mathrm{Pb}$ collisions as a function of associated particles $p_{\mathrm{T}}$.

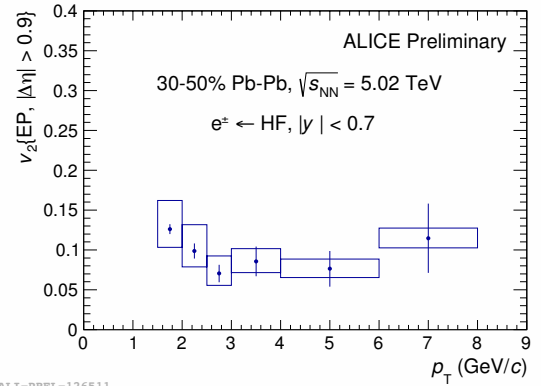

Figure 5: Elliptic flow $\left(v_{2}\right)$ for $e^{\mathrm{HF}}$ in semicentral $(30-50 \%) \mathrm{Pb}-\mathrm{Pb}$ collisions at $\sqrt{s_{\mathrm{NN}}}=$ $5.02 \mathrm{TeV}$.

in semi-central (30-50\%) in $\mathrm{Pb}-\mathrm{Pb}$ collisions at $\sqrt{s_{\mathrm{NN}}}=5.02 \mathrm{TeV}$ [9] (Fig. 5). The positive $v_{2}$ of $\mathrm{e}^{\mathrm{HF}}$ suggests that heavy-flavour quarks participate to the collective motion of the system. The $R_{\mathrm{AA}}$ 
and $v_{2}$ for $e^{\mathrm{HF}}$ are reproduced by pQCD-based energy loss and transport models [10-15] although the simultaneous description of the $R_{A A}$ and $v_{2}$ is challenging as shown in Fig. 6.
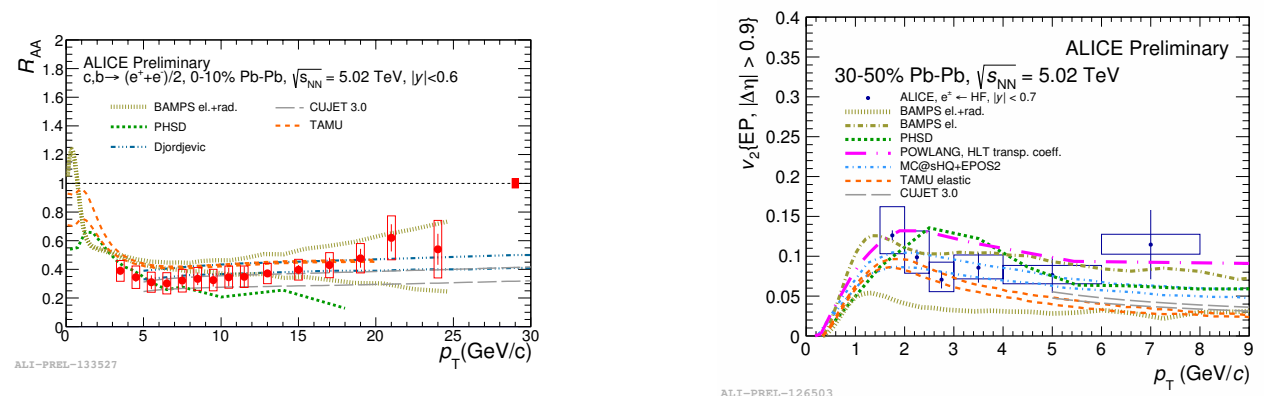

Figure 6: Transverse momentum dependence of $R_{\mathrm{AA}}$ in $0-10 \%$ (left) and $v_{2}$ in $30-50 \%$ (right) of $\mathrm{e}^{\mathrm{HF}}$ compared with expectations from theoretical models [10-15].

\section{Summary}

The ALICE experiment measured several differential observables in various collision systems and energies, with the goal of understanding the interaction of heavy quarks with the constituents of the QGP formed in $\mathrm{Pb}-\mathrm{Pb}$ collisions. The production of $\mathrm{D}$-tagged jet in pp collisions and the azimuthal correlation of $\mathrm{D}$ mesons with charged particles in $\mathrm{p}-\mathrm{Pb}$ collisions are consistent with expectations from PYTHIA and POWHEG+PYTHIA event generators. A strong suppression and positive $v_{2}$ of leptons from semi-leptonic decays of heavy-flavour hadrons is observed in $\mathrm{Pb}-\mathrm{Pb}$ collisions at $\sqrt{s_{\mathrm{NN}}}=5.02 \mathrm{TeV}$. Those results suggest significant energy loss of charm and beauty quarks in the hot and dense QCD matter and their participation to the collective motion of the system as a result of strong interaction with medium constituents.

\section{References}

[1] M. Djordjevic et al. Phys. Lett. B, 632 (2006) 81

[2] A. Adil, I. Vitev, Phys. Lett. B, 649 (2007) 139

[3] B. Abelev et al. (ALICE collaboration), JHEP 01 (2012) 128

[4] N. Armesto, J. Phys. G, 32 (2006), pp. 367-394

[5] J. W. Cronin et al. Phys. Rev. D, 11 (1975), p. 3105

[6] Z.-B. Kang, I. Vitev, E. Wang, H. Xing, C. Zhang, Phys. Lett. B, 740 (2015), pp. 23-29

[7] K. Aamodt et al. (ALICE collaboration), Phys. Rev. Lett. 108, 092301

[8] J. Adam et al. (ALICE collaboration), Phys. Lett. B763, pages238-250

[9] D. Godoy for the Alice collaboration, Nuclear Physics, Section A 967C (2017) pp. 636-639

[10] A. Beraudo, A. De Pace, M. Monteno, M. Nardi, F. Prino, Eur. Phys. J. C75 (2015) 121.

[11] M. Nahrgang, J. Aichelin, P. B. Gossiaux, K. Werner, Phys. Rev. C89 (2014) 014905.

[12] J. Upho, F. Senzel, O. Fochler, C. Wesp, Z. Xu, C. Greiner, Phys. Rev. Lett. 114 (2015) 112301.

[13] M. He, R. J. Fries, R. Rapp, Physics Letters B 735 (2014) 445-450.

[14] M. Djordjevic, M. Djordjevic, Phys. Rev. C92 (2015) 024918.

[15] T. Song, H. Berrehrah, D. Cabrera, W. Cassing, E. Bratkovskaya, Phys. Rev. C93 (2016) 034906. 